\title{
Upward Spirals of Mindfulness and Reappraisal: Testing the Mindfulness-to-Meaning Theory with Autoregressive Latent Trajectory Modeling
}

\author{
Eric L. Garland ${ }^{1} \cdot$ Laura G. Kiken ${ }^{2} \cdot$ Kim Faurot $^{3} \cdot$ Olafur Palsson $^{3}$ • \\ Susan A. Gaylord ${ }^{3}$
}

Published online: 12 March 2016

\begin{abstract}
The mindful reappraisal hypothesis of the Mindfulness-to-Meaning Theory (Garland et al. in Psychol Inquiry 26(4):293-314, 2015a; Psychol Inquiry 26(4): 377-387, 2015b) proposes that mindfulness generates eudaimonic well-being by promoting positive reappraisal, the positive psychological process through which stressful events are re-construed as benign, meaningful, or growthpromoting. To test this hypothesis, we examined prospective relations between state mindfulness and positive reappraisal in a community sample participating in a mindfulness-based intervention (MBI). At seven weekly time points throughout the MBI, participants $(\mathrm{N}=234)$ engaged in a 10-min mindfulness meditation exercise at home and completed a measure of the degree of state mindfulness experienced during the meditation, as well as a measure of their use of positive reappraisal over the previous week. Support for the mindful reappraisal hypothesis of the Mindfulness-to-Meaning Theory was found: in latent growth curve and multivariate autoregressive latent trajectory models, increases in the trajectory of state mindfulness experienced during meditation were significantly and robustly associated with more frequent use of positive reappraisal over the course of participation in the 8 weeklong MBI. Thus, mindfulness and reappraisal may
\end{abstract}

Eric L. Garland

elgarlan@gmail.com

1 University of Utah, 395 South, 1500 East, Salt Lake City, UT 84112, USA

2 Kent State University, Kent, OH, USA

3 University of North Carolina at Chapel Hill, Chapel Hill, NC, USA reciprocally enhance one another as interdependent components of a positive feedback loop whose structure might be best described as an upward spiral.

Keywords Mindfulness - Reappraisal - Upward spiral · Positive psychology $\cdot$ Emotion regulation · Mindfulness-toMeaning

\section{Introduction}

The rise of mindfulness-based interventions (MBIs) in medicine and psychology may be due, in part, to their potential to enhance eudaimonic well-being (Brown and Ryan 2003), yet, the scientific literature has largely focused on the efficacy of MBIs for reducing various forms of psychological distress, including anxiety and depression (Goyal et al. 2014; Khoury et al. 2013; Hofmann et al. 2010). Unlike hedonic approaches to well-being, which depend on obtaining pleasure and avoiding pain, eudaimonic well-being is characterized by a sense of meaning and purposeful, positive engagement with life even under conditions of adversity (Ryan and Deci 2001). Psychometric evidence suggests that these two forms of well-being are distinct yet interrelated constructs: hedonic well-being is correlated with basic emotional states like elevated positive affect and reduced negative affect, whereas eudaimonic well-being converges with higher-order cognitive constructs like self-realization and autonomy (Ryff and Singer 2008). Recent research also suggests that the construct of mindfulness is associated with both hedonic and eudaimonic well-being, with closer links to eudaimonia (Hanley et al. 2015).

The notion that mindfulness may enhance eudaimonic well-being does not frequently appear in standard 
operationalizations of mindfulness, which define mindfulness as the practice of cultivating non-judgmental attention to present moment experience without clinging to positive experience or avoiding negative experience (Kabat-Zinn 1990). As such, most scientific models of mindfulness offer a primarily eliminative account of how mindfulness produces therapeutic effects, focusing on the extinction of negative states of mind rather than the generation of eudaimonic states of mind.

In contrast to such eliminative models, recent theorizing posits that mindfulness may stimulate eudaimonic wellbeing by promoting positive psychological processes. One such generative account, called the Mindfulness-to-Meaning Theory (Garland et al. 2015a) proposes that mindfulness generates eudaimonic meaning primarily through the mechanism of positive reappraisal - the adaptive process through which stressful events are re-construed as benign, meaningful, or even growth-promoting (Lazarus and Folkman 1984). According to the Mindfulness-to-Meaning Theory, the practice of mindfulness evokes a metacognitive, flexible state of awareness which suspends habitual appraisal tendencies via decentering and broadens attention to encompass an expanded set of contextual information from which reappraisals can be generated. Further, positive reappraisal conceivably may promote a mindset that is favorable for mindfulness. For example, as posited in a precursor to the Mindfulness-to Meaning Theory (Garland et al. 2011), because reappraisal may involve a similar set of top-down cognitive control mechanisms as utilized in mindfulness (e.g., attentional orienting, inhibitory control, etc.), it may increase the likelihood of nonjudgmental attention to the present moment (also see Garland et al. 2015b). In the Mindfulness-to-Meaning Theory, these proposed reciprocal relations between mindfulness and positive reappraisal-termed the mindful reappraisal hypothesis - are central to a larger upward spiral process leading toward eudaimonic well-being. Therefore, an important part of the theory suggests that during an MBI, increases in mindfulness over time (Kiken et al. 2015) should be accompanied by increases over time in the use of positive reappraisal, and that these two growth processes may be mutually reinforcing.

A number of cross-sectional, observational, and experimental studies support relations between mindfulness and reappraisal, though they do not examine how these constructs may develop together over time. For example, cross-sectional research shows that dispositional mindfulness is significantly associated with reappraisal among healthy individuals and those with psychiatric, medical, and substance use disorders (Desrosiers et al. 2013; Garland et al. 2014c; Hanley and Garland 2014). Participation in MBIs has been shown to be associated with significant increases in positive reappraisal relative to non-randomized comparison groups (Huston et al. 2011; Jones and Hansen 2014), and prospective observational research demonstrates that reappraisal statistically mediates the stress reductive effects of increasing dispositional mindfulness through MBIs (Garland et al. 2011). Laboratory research indicates that individuals who completed a MBI evidenced significantly greater positive reappraisal ability during an experimental sad mood induction than a matched control group or those who had been treated with cognitive-behavioral therapy (Troy et al. 2012). In an experimental study of brief mindfulness training, the degree of state mindfulness achieved during the act of mindfulness meditation was prospectively and positively associated with increases in reappraisal during the following week; path analysis revealed that the indirect effect between brief mindfulness training and reappraisal was significant through state mindfulness (Garland et al. 2015e). Lastly, a longitudinal, randomized controlled trial of MindfulnessOriented Recovery Enhancement (MORE), an intervention which explicitly teaches mindfulness skills as a means of enhancing reappraisal, indicated that chronic pain patients assigned to 8 weeks of MORE had significantly greater increases in positive reappraisal than those in a social support group (Garland et al. 2014b). Findings across these varied study samples, research designs, and measurement approaches suggest the presence of a fundamental relation between mindfulness and positive reappraisal, providing support for the Mindfulness-to-Meaning Theory. Yet, the aforementioned studies did not examine potential relations between processes of change in these constructs over time during an MBI. Most only assessed the association between mindfulness and reappraisal at one or two time points, without considering a time-dependent process in which these constructs might develop together and contribute to each other. A longitudinal study with multiple repeated measures is needed to reveal temporally-dynamic changes in mindfulness and reappraisal.

In response to this need, the present study aimed to test the mindful reappraisal hypothesis of the Mindfulness-toMeaning Theory via a repeated measures design that could assess time-lagged associations between state mindfulness and positive reappraisal among a sample of individuals participating in a mindfulness-based stress and pain management course. Our primary study hypothesis (hypothesis 1) was that the trajectories of state mindfulness and positive reappraisal would be positively associated over time in multivariate latent growth curve models that account for the temporal dynamics of these putatively interrelated phenomena. Further specifying the presumed dynamical relation between state mindfulness and reappraisal over time, we employed multivariate autoregressive latent 
trajectory modeling to test the following hypotheses integral to the upward spiral posited by the Mindfulness-toMeaning Theory: a) (hypothesis 2-autoregressive component of state mindfulness) elevations in state mindfulness on a given week will predict elevations in state mindfulness on the following week, above and beyond any trait-like propensity toward state mindfulness; b) (hypothesis 3autoregressive component of positive reappraisal) elevations in positive reappraisal on a given week will predict elevations in positive reappraisal on the following week, above and beyond any trait-like propensity toward positive reappraisal; and c) (hypothesis 4-cross-lagged relations between state mindfulness and positive reappraisal) elevations in state mindfulness on a given week will predict elevations in positive reappraisal on the following week, and vice versa.

\section{Method}

The study employed a prospective, observational design with weekly repeated measures over the course of a mindfulness-based intervention (MBI).

\section{Participants}

Participants were recruited from an 8-week mindfulness program based on MBSR (Kabat-Zinn 1990) and offered several times each year by the University of North Carolina Program on Integrative Medicine. Sessions correspond with the MBSR curriculum in terms of mindfulness techniques and mindfulness principles discussed. Like MBSR, this MBI provided weekly training in mindful breathing and body scan meditations, with additional practice of mindful walking, yoga, and lovingkindness meditation in later weekly sessions. Sessions were approximately $2 \mathrm{~h}$ long. There was a half-day meditation retreat as well. Participants were asked to practice mindfulness skills for approximately $45 \mathrm{~min}$ per day. Trainers had completed MBSR teacher training. Prior to the start of the intervention, program participants were invited to enroll in the research study for a $\$ 25$ reduction in the program fee. Participants were eligible for the study if they were $\geq 18$ years old, fluent in English, and able to complete repeated surveys online. The sample of consisted of $N=234$ participants who enrolled in the MBI. Sample demographics indicated that the majority $(75 \%)$ of participants were female and were predominately white $(83 \%)$, with a mean age of 44.83 $(S D=14.32)$. Approximately two-thirds of the sample $(62 \%)$ had graduate degrees, and $60 \%$ had a household income $>\$ 60,000$. More than half $(57 \%)$ of participants completed at least four weekly assessments.

\section{Measures}

\section{State Mindfulness}

The Toronto Mindfulness Scale (TMS; Lau et al. 2006) is a 13-item instrument assessing state mindfulness, with good psychometric properties. Items are rated on a 5-point scale ranging from 0 (not at all) to 4 (very much). Higher total scores indicate higher overall state mindfulness (range in the current sample $=0-52$ ). Participants were asked to use this measure to describe their mental state during a 10-min meditation session (see Procedures). In the current study, the TMS showed a high degree of internal consistency across all 7 weeks of measurement $(\alpha=.90-.95)$.

\section{Positive Reappraisal}

Habitual use of reappraisal was measured with the 4-item positive reappraisal subscale of the Cognitive Emotion Regulation Questionnaire (CERQ) (Garnefski and Kraaij 2007), an internally-consistent subscale which asked the respondent how often they "think I can become a stronger person as a result of what has happened" or "look for positive sides to the matter" to cope with stressful events over the past week. Responses are rated on a scale ranging from 1 (almost never) to 5 (almost always); a reappraisal total score can be obtained by taking the average of the four items (range in the current sample $=1-5$ ). In prior research, scores on this reappraisal scale were prospectively predictive of lower levels of future affective symptoms (Garnefski and Kraaij 2007), and changes in CERQ reappraisal scores mediated the stress-reductive effects of mindfulness (Garland et al. 2011). In this study, the CERQ reappraisal subscale showed a high degree of internal consistency across all 7 weeks of measurement $(\alpha=.92$ $.95)$.

\section{Procedure}

Instructions for research participation were first provided at the MBI program orientation session, a week before the start of the intervention. After each weekly class during the MBI, participants received an emailed link to an online survey. Participants were instructed to first complete a 10-min mindfulness meditation session using a technique learned in the intervention (i.e., body scan or mindful breathing). Immediately after the mindfulness meditation, participants were presented with the TMS and asked to use this scale to rate their experience during that particular meditation session, and then were presented with the CERQ and asked to use this scale to rate their habitual use of positive reappraisal during the previous week. 


\section{Statistical Analyses}

Preliminary analyses assessed bivariate correlations between variables. Additionally, we used multilevel, linear mixed models with maximum likelihood estimation of missing data to examine growth in mindfulness and positive reappraisal over the study period.

To test the primary hypotheses, we employed multivariate latent growth curve (LGC) modeling. Multivariate LGC modeling was well-suited to our interests because can assess variability in individuals' trajectories of change in two variables over time, and how these two change trajectories inter-relate. In a basic LGC model, a latent variable is used to represent individual (and potentially variable) trajectories of change on a measure over time. Paths from this latent trajectory variable to the repeated observed scores are used to indicate the rate of time. If trajectories of change are linear, then they can be represented as a latent slope variable that loads onto the repeated observed scores with a constant increment of change (e.g., change per week over seven weekly measures, with factor loadings set to $0,1,2,3,4,5$, and 6 to account for this change). Preliminary testing with our data confirmed that a latent slope model fit the data better than curvilinear trajectories. In addition to the latent trajectory/slope variable, a separate latent variable represents individual intercepts, or estimated initial status. The intercept factor was defined such that the factor loadings for all repeated measures were fixed to 1 . The intercept factor can be interpreted to index the trait-like aspects of the propensity towards achieving states of mindfulness and the habitual tendency to use positive reappraisal as a coping strategy. Multivariate LGC additionally tests for the covariation between latent intercept and slope factors between variables of interest-in this case, state mindfulness and positive reappraisal.

This multivariate LGC model was the first model tested, Model 1. Beyond overall model fit, of interest in Model 1 was the variance of the latent slopes of state mindfulness and positive reappraisal. The variance parameter indicated the degree of individual variability in the rate of change in state mindfulness and positive reappraisal over the course of the MBI.

Secondary study hypotheses were tested with a series of autoregressive latent trajectory (ALT) growth models (Bollen and Curran 2004). ALT models combine features of a LGC model with potential autoregressive and crosslagged effects. Though other modeling approaches were possible (e.g., latent difference score models), this ALT modeling strategy allowed us to simultaneously estimate the time-specific autoregressive and cross-lagged effects of weekly state mindfulness and positive reappraisal on subsequent weekly levels, while accounting for stable, traitlike trajectories in these variables over time. Thus, ALT models provide a robust means of concurrently assessing the respective influence of trait-like stability and state-like change. In ALT modeling, a model building process is used whereby a series of increasingly more complex models are tested leading to the testing of a full model with both crosslagged and autoregressive parameters. These models may yield different results as parameters are added and removed from successive models. The relative parsimony of these models is then tested through comparison of fit statistics.

A multivariate ALT model was tested to examine whether state mindfulness and positive reappraisal reciprocally preserve and energize one another from week to week in an upward spiral over time (Garland et al. 2010). Autoregressive parameters were estimated by regressing a given week's level of state mindfulness and positive reappraisal on the prior week's levels of these constructs, whereas cross-lagged pathways were estimated by testing the relation between the previous week's level of state mindfulness and following week's level of positive reappraisal and vice versa. For purposes of parsimony, we constrained autoregressive and cross-lagged parameters to equality when such constraints did not produce significant decrements in model fit. Though we could have modeled mindfulness and reappraisal as latent variables at each time point, the multivariate ALT models we ran were already so complex in terms of the number of statistical parameters estimated that we opted to simplify our model. This choice of treating mindfulness and reappraisal as observed rather than latent variables was also warranted given the very high internal consistencies ( $\alpha$ 's $>.90)$ observed for the mindfulness and reappraisal scales. A similar decision was made with regard to specifying the shape factors of latent slope variables in the ALT models. In the interest of parsimony, we ultimately selected a linear slope shape, because freely estimating slope loadings did not significantly improve model fit, nor did it substantially change the valence or significance of theoretically-relevant model parameters.

Amos Version 19.0 was used to estimate all ALT models. To handle missing data, the LGC and ALT models under investigation employed full information maximum likelihood estimation, which uses all available information from partially missing cases in analyses. Data points from all study cases were included in these models $(\mathrm{N}=234)$. Model fit was evaluated based on the Chi square statistic (Kline 1998), as well as with the comparative fit index (CFI: Bentler 1990) and the root mean squared error of approximation (RMSEA; Browne and Cudeck 1993). In addition to the presence of a non-significant Chi square ratio, adequate model fit was indicated by CFI values exceeding .90 and RMSEA values less than .08. Model comparisons were performed using a Chi square difference test. 


\section{Results}

Inter-correlations and descriptive statistics, including means and standard deviations, are shown in Table 1. Linear mixed models indicated that slope of state mindfulness was significant, indicating that state mindfulness significantly increased over time, $F(6,110.43)=14.83$, $p<.001$, Hedge's $g=1.01$. Similarly, the slope of reappraisal was significant, indicating that reappraisal significantly increased over time, $F(6,119.04)=5.30, p<.001$, Hedge's $g=.58$.

\section{Model 1 (Hypothesis 1): Was There a Positive Correlation Between the Slopes of State Mindfulness and Positive Reappraisal During the Intervention?}

The multivariate LGM (Fig. 1) fit the data well, $X^{2}=87.27, d f=74, p=.14 ; \mathrm{CFI}=.99 ;$ RMSEA $=.03$ (95\% CI .00, .05). To explicate the relation between state mindfulness and positive reappraisal trajectories, the covariance parameter between the two latent slope factors was assessed. Providing support for our first hypothesis, the state mindfulness and positive reappraisal latent slope factors were robustly positively correlated $(r=.68$, $p<.001$ ), indicating that individuals who experienced the greatest increases in state mindfulness during meditation also reported the largest weekly increases in positive reappraisal over the course of the MBI. Of note, the state mindfulness and positive reappraisal latent intercept factors were also significantly positively correlated $(r=.58$, $p<.001$ ).

In addition, significant variances for the latent intercept factors $(p s<.001)$ of state mindfulness and positive reappraisal indicated that individuals varied in their baseline status on state mindfulness and positive reappraisal. There was also significant variability in the slope of state mindfulness $(M=2.43, S E=.53 ; p<.001)$. That is, while on average state mindfulness increased by about $17 \%$ over the course of the MBI, the rate of increase in state mindfulness was greater for some individuals than for others (including no change for some). There was a small yet statistically significant inverse association between the slope and intercept of state mindfulness $(r=-.29$, $p=.04)$, indicating that individuals with higher baseline levels of state mindfulness reported less steep increases in state mindfulness over time. Similarly, there was also significant variability in the slope of positive reappraisal $(M=.01, S E=.004 ; p<.001)$. That is, while on average positive reappraisal increased by about $30 \%$ over the course of the MBI, the rate of increase in positive reappraisal was greater for some individuals than for others (including no change for some). The slope and intercept of positive reappraisal did not significantly covary, indicating no relation between individuals' estimated baseline status of positive reappraisal use and their rate of change.

\section{Model 2 (Hypotheses 2 and 3): Were There Autoregressive Effects for State Mindfulness and Positive Reappraisal?}

This model (Fig. 2) fit the data well, $\chi^{2}=73.91, d f=71$, $p=.38$; CFI $=1.00$; RMSEA $=.01(95 \%$ CI $.00, .04)$. There was a significant autoregressive parameter for positive reappraisal, $\mathrm{b}=.15(\mathrm{SE}=.04), p=.005$, such that the propensity toward using positive reappraisal during the previous week influenced the tendency to use positive reappraisal the following week. However, contrary to our hypothesis, the autoregressive parameters for state mindfulness were non-significant. As in the LGM (Model 1), the latent slopes $(r=.90, p<.001)$ and intercepts $(r=.62$, $p<.001)$ of state mindfulness and positive reappraisal were significantly and robustly positively correlated.

\section{Model 3 (Hypotheses 4): Were There Cross-Lagged Effects Between State Mindfulness and Positive Reappraisal?}

This model also fit the data well, $\chi^{2}=81.20, d f=72$, $p=.21$; CFI $=.99$; RMSEA $=.02(95 \%$ CI $.00, .05)$. This model contained cross-lagged effects such that week 2 positive reappraisal was regressed on week 1 state mindfulness, and week 2 state mindfulness was regressed on week 1 positive reappraisal, etc. This cross-lagged pattern was estimated across all 7 weeks. Estimates indicated the presence of significant cross-lagged effects, such that increases in state mindfulness over a given week predicted increased use of positive reappraisal on the following week, $\mathrm{b}=.01$ (SE $=.005), p=.002$. However, in this model, the cross-lagged parameters from positive reappraisal to state mindfulness were non-significant, $b=.73$ $(\mathrm{SE}=.41), p=.07$. While the latent intercepts of state mindfulness and positive reappraisal were significantly positively correlated $(r=.48, p<.001)$, in this model there was no significant association between latent slope factors.

\section{Model 4 (Full Multivariate ALT Model)}

The full multivariate ALT model with both autoregressive and cross-lagged effects (Fig. 3) also fit the data well: $\chi^{2}=73.89, d f=70, p=.35 ; \mathrm{CFI}=1.00 ;$ RMSEA $=.02$ (95\% CI .00, .04). In this model, significant autoregressive effects were observed for weekly changes in positive reappraisal, $\mathrm{b}=.14(\mathrm{SE}=.06), p=.01$, and cross-lagged effects were observed such that increases in positive 


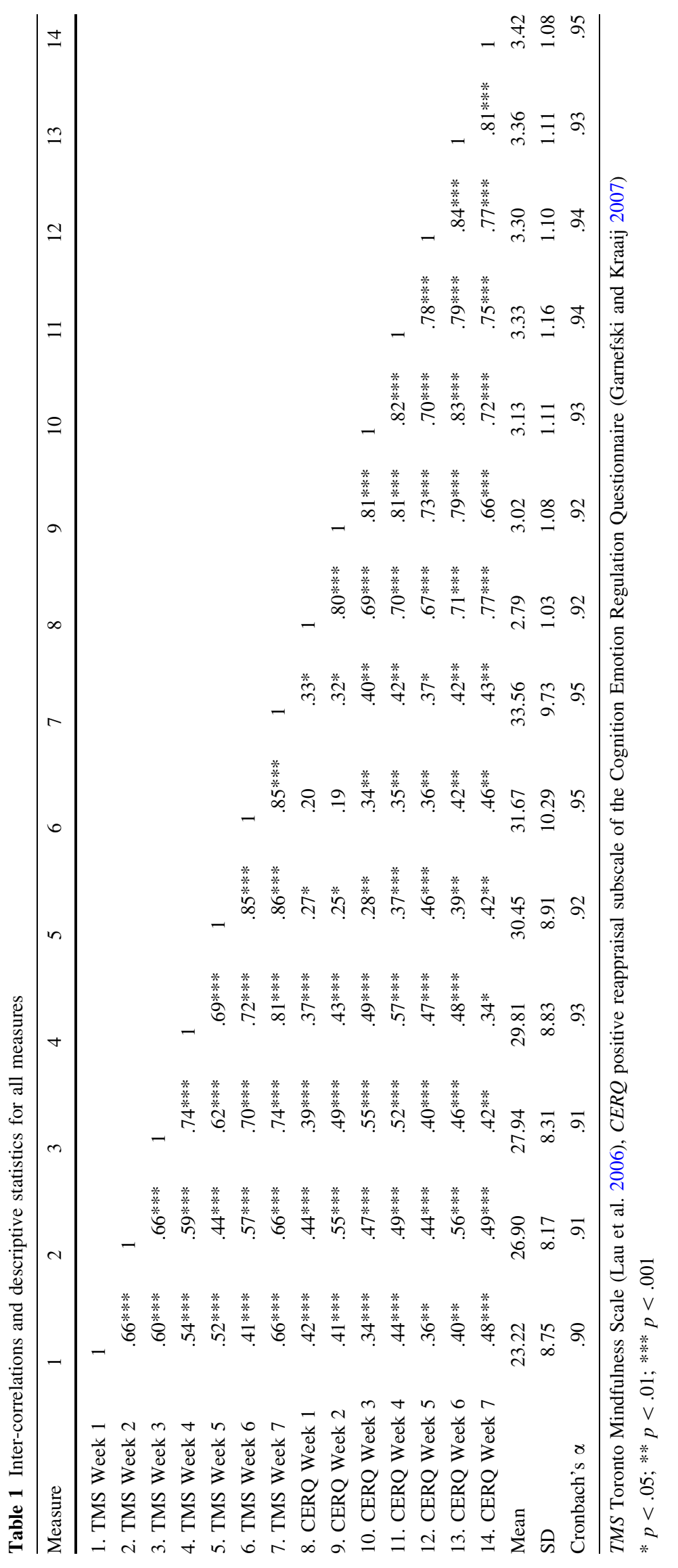




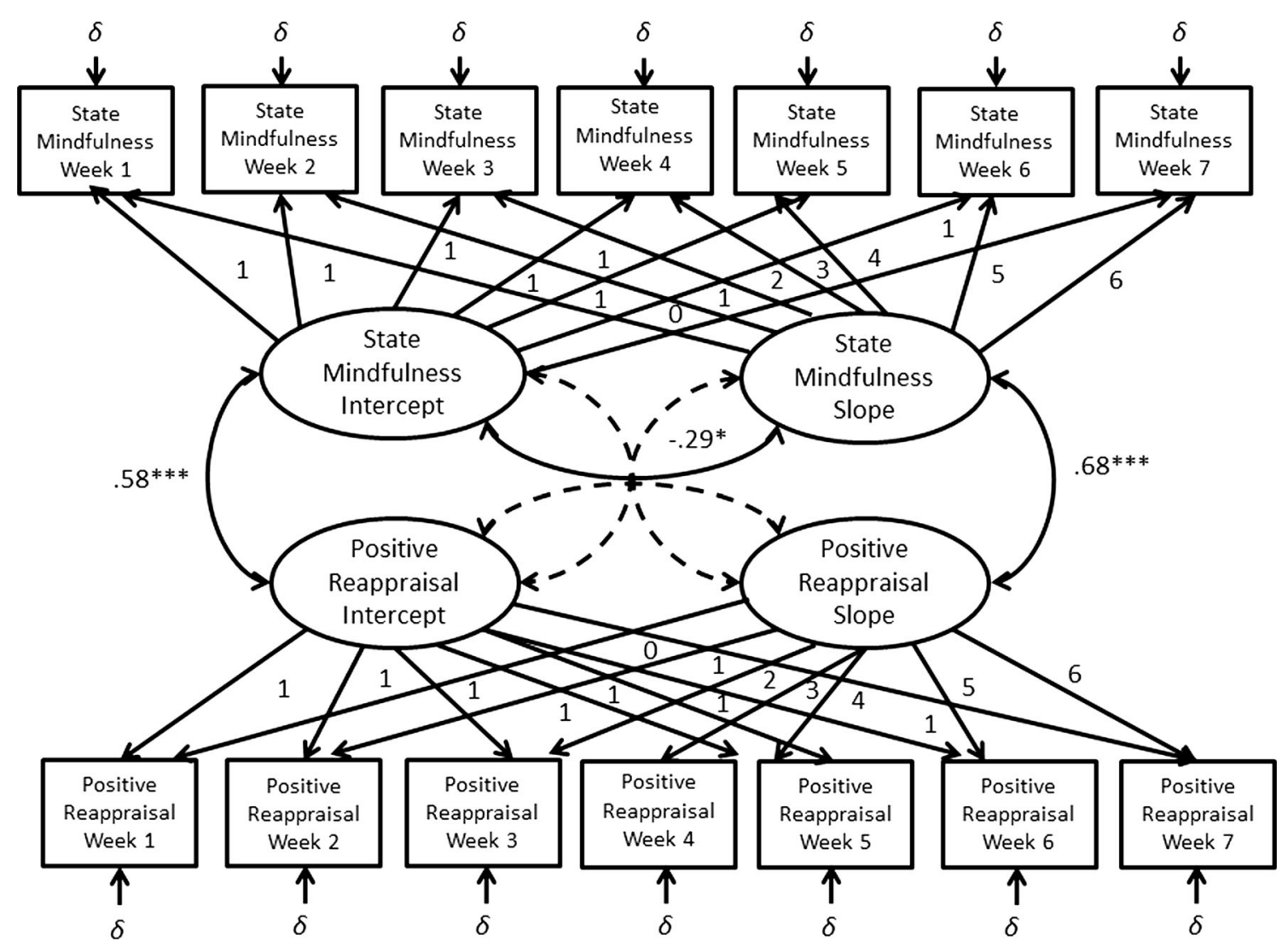

Fig. 1 Latent growth curve model of weekly changes in state mindfulness and positive reappraisal over an 8-week mindfulness-based intervention

reappraisal over a given week predicted increased state mindfulness over the following week, $\mathrm{b}=.99(\mathrm{SE}=.47)$, $p=.04$. However, increased state mindfulness did not significantly predict the following week's use of positive reappraisal, nor were there autoregressive effects observed for state mindfulness in this model. Both the latent intercepts $(r=.48, p<.001)$ and latent slopes $(r=.58$, $p<.001)$ of state mindfulness and positive reappraisal were significantly positively correlated in this model.

\section{Model Comparison}

For the purposes of parsimony, the full multivariate ALT model (Model 4) was compared to Models 2 (autoregressive only) and 3 (cross lags only) using the Chi square difference test. Constraining the full ALT model to a LGC model with only autoregressive effects did not lead to a significant decrement in model fit, $\chi^{2}$ change $=.03$, $d f=1, p=.86$, whereas constraining the full ALT model to a LGC model with only cross-lagged effects did result in a significant difference in model fit, $\chi^{2}$ change $=7.31$, $d f=2, p=.02$. Thus, Model 2 (LGM with autoregressive effects) was the most parsimonious model tested.

\section{Discussion}

As a whole, study findings provide support for the mindful reappraisal hypothesis of the Mindfulness-to-Meaning Theory. In multivariate latent growth curve analyses, increases in the trajectory of state mindfulness experienced during meditation were associated with more frequent use of positive reappraisal coping over the course of participation in an 8 week-long MBI. Notably, the relation between these two trajectories was strong and significant across all tested models, even in models that accounted for significant autoregressive effects for weekly changes in reappraisal. In one of our analysis models, evidence was also found for cross-lagged relations in which the degree of state mindfulness achieved during the practice of mindfulness meditation predicted the extent to which a practitioner would engage in positive reappraisal coping in the following week, and greater use of positive reappraisal coping predicted increased levels of state mindfulness in successive weeks. Thus, considering the consistently significant association between trajectories of mindfulness and reappraisal across all analysis models, as well as the indication of cross-lagged effects, mindfulness and 


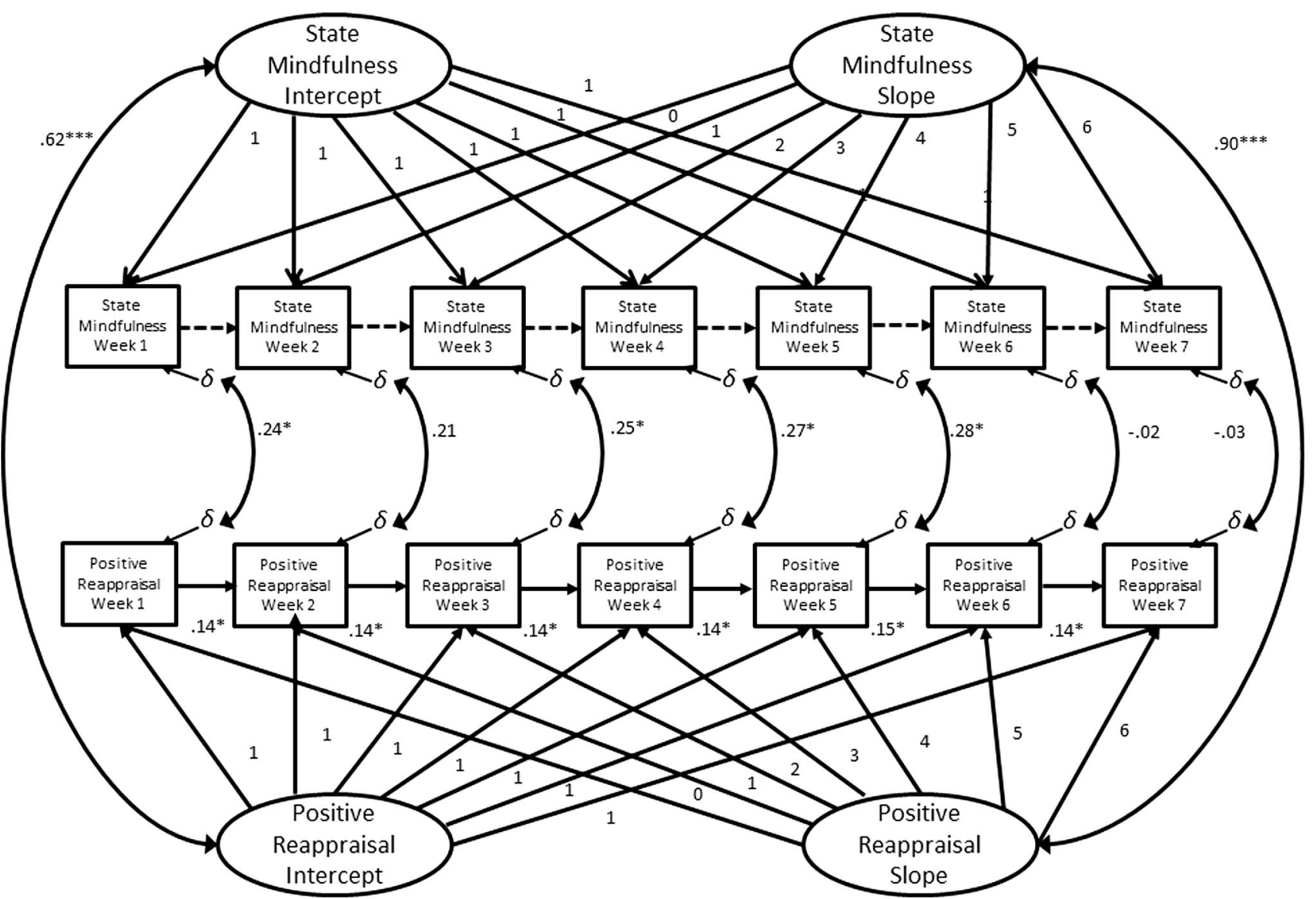

Fig. 2 Autoregressive latent trajectory model of weekly changes in state mindfulness and positive reappraisal over an 8-week mindfulness-based intervention

reappraisal might be viewed as interdependent components of a positive psychological system whose structure may be described as an upward spiral - that is, a dynamic process in which states of mindfulness experienced during the act of meditation and positive reappraisal habits mutually reinforce and strengthen one another over time.

In ALT models, both mindfulness and positive reappraisal were found to exhibit trait-like properties, suggesting that a significant portion of the variance in each factor as it was expressed from week to week was a function of an underlying dispositional propensity toward experiencing the state of mindfulness and the habitual use of reappraisal, respectively. Yet, study findings also suggest the presence of state-like, time-specific effects whereby instances of state mindfulness and weekly use of reappraisal inter-relate and potentially support one another over time. The presence of significantly covarying latent growth factors indicates that irrespective of the trait-like propensity towards mindfulness and reappraisal, and beyond any effect of transitory mindful states and instances of reappraisal use, an increasing trajectory in one of these factors over the course of the MBI was closely intertwined with the other. Thus, experiencing higher levels of state mindfulness and increasing one's use of reappraisal from week-to-week may engender a self-reinforcing cycle during the process of mindfulness training. These findings might be interpreted as evidence for a positive feedback loop, in which states of mindfulness may serve as system inputs to amplify positive reappraisal habits, which in turn increase the likelihood that that practitioner will experience mindful states in the future. To support this claim, the temporal ordering of these constructs is required, yet crosslagged effects were inconsistent across models, with Model 3 demonstrating cross-lagged effects from state mindfulness to reappraisal, and Model 4 demonstrating cross-lagged effects from reappraisal to mindfulness, but not vice versa. Model inconsistencies might stem from the modest strength of cross-lagged effects. Alternatively, it is very likely that the reciprocal effect from mindfulness to reappraisal occurs at a smaller time scale, from one day to the next, or within a given emotion regulatory episode-as specified in the Mindfulness-to-Meaning Theory (Garland et al. 2015a, b). To examine this hypothesis, more frequent observations (e.g., via experience sampling or ecological momentary assessment) over the course of mindfulnessbased treatment are needed. 


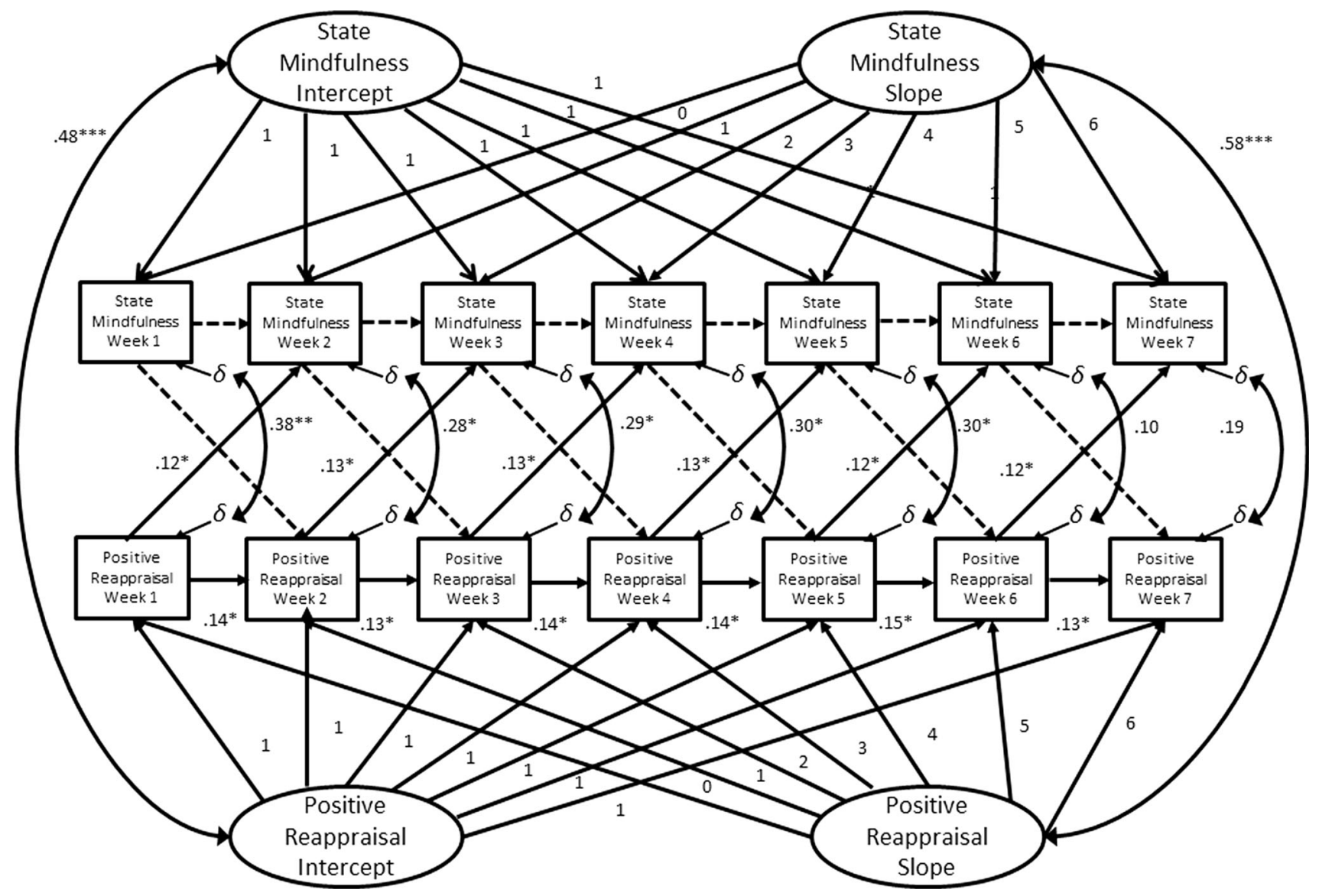

Fig. 3 Latent trajectory model with autoregressive and cross-lagged effects between changes in state mindfulness and positive reappraisal over an 8-week mindfulness-based intervention

Study findings might be partly explicated via a cognitive capacity account, in that repeatedly activating the cognitive processes integral to both reappraisal and mindfulness (attentional re-orienting, holding information online in working memory, set-shifting, metacognition, etc.) may promote these domain general resources that could then be utilized to access the state of mindfulness or to use reappraisal (Garland et al. 2015b). In support of this interpretation, recent neuroimaging analysis indicate that both mindfulness and reappraisal of negative emotional stimuli recruit overlapping brain regions known to be involved in emotion regulation, including the dIPFC and vmPFC (Opialla et al. 2014). Thus, reappraisal may exercise similar brain circuits to those that are involved in mindfulness, ${ }^{1}$ and therefore more frequent use of either skill may enhance the likelihood and efficacy of the other.

\footnotetext{
1 Though mindfulness and reappraisal may draw on a similar subset of neural resources among novices, among adept meditation practitioners mindfulness may be decidedly less effortful, and therefore rely on comparatively less prefrontal cortical activation than reappraisal (Hölzel et al. 2011).
}

Considering broader processes, the Mindfulness-toMeaning Theory (Garland et al. 2015a, b) proposes a larger upward spiral process leading toward eudaimonic wellbeing. Though we tested proximal psychological processes that might lead to eudaimonia, no distal measures of eudaimonic well-being were available-limiting our ability to fully test the model. In addition, the Mindfulness-toMeaning Theory includes several additional variables not tested in the present research which may support reciprocal relations between mindfulness and reappraisal, such as disrupted automatized scripts and schemas, cognitive flexibility, positive emotions, and attention to positive information. Moreover, due to its focus on testing a specific hypothesis of the Mindfulness-to-Meaning Theory, the present study also omitted constructs that are outside of the theory yet logically might also be linked with mindfulness and reappraisal (e.g., self-compassion).

In that regard, the upward spiral dynamic posited by the Mindfulness-to-Meaning Theory parallels a recent analysis of experience sampling data from a RCT of MindfulnessBased Cognitive Therapy (MBCT) for patients with a history of depression (Garland et al. 2015d), which found 
that mindfulness training stimulated autoregressive and cross-lagged effects between positive cognitive and affective processes as revealed by a multivariate ALT model similar to the ones tested in the current study. Moreover, MBCT appeared to strengthen the cross-lagged relationship between current positive affect and positive cognition on the following day, but not the cross-lagged relation between current positive cognition and subsequent positive affect, suggesting that for those individuals who respond to mindfulness training by experiencing enhanced positive affect, these enhancements tend to maintain themselves through a self-reinforcing cycle that is largely impelled by emotion. It is possible that including positive affect in the present models would have revealed more consistent support for weekly cross-lagged relations between mindfulness and reappraisal. Unfortunately, no measure of positive affect was available in the present study.

We also could not assess the second fundamental postulate of the Mindfulness-to-Meaning Theory, which may be relevant for fully understanding the upward spiral process: The mindful savoring hypothesis suggests that mindfulness promotes the ability to savor and experience reward from positive daily experiences. Prior RCTs of MBIs have provided support for this hypothesis by demonstrating significant effects of mindfulness training on enhancing natural reward processing (Garland et al. 2014a, 2015c; Geschwind et al. 2011). Future studies should include measures of both positive cognitive (e.g., reappraisal) and positive affective processes (e.g., savoring) to provide a more complete test of the theory.

The present study was also limited by its prospective, observational design. Although we assessed time-lagged relations between mindfulness and reappraisal, study findings were essentially correlational in nature. To determine the causal influence of mindfulness on reappraisal, experimental studies are needed in which meditation-naïve participants are taught basic mindfulness practices (e.g., mindful breathing) and compared to a no-meditation control group with regard to their positive reappraisal ability (e.g., Garland et al. 2015e). We also relied on self-reports, which may be subject to biases. Furthermore, the TMS is designed to measure the acute state of mindfulness achieved during a meditation session, whereas the CERQ measures the habitual (or trait-like) use of positive reappraisal coping. These differences in measurement timeframe may have also affected our statistical estimates of cross-lagged and autoregressive parameters. Also, we did not assess meditation practice frequency-which may have influenced the relation between state mindfulness and reappraisal. Investigations which employ a RCT design, behavioral tasks (e.g., an emotion regulation task in which participants are asked to reappraise negative emotional images or savor positive emotional images), and psychophysiological methods (e.g., analysis of heart rate variability or the late positive potential (LPP) component of the electroencephalogram) would allow for a more robust test of the Mindfulness-to-Meaning Theory.

In the present study, participants in an 8-week mindfulness-based training course increased their use of positive reappraisal. This is notable given reappraisal was not the focus of the course. Yet, the present findings suggest that intentionally combining mindfulness practice with training in positive reappraisal would significantly enhance reappraisal ability. Study results have implications for a psychotherapeutic approach that unites mindfulness training with cognitive-behavioral therapy (CBT) and principles from positive psychology. While meditation practice may temporarily interrupt narrative-evaluative processing of stressors when the state of mindfulness is achieved, inevitably once the meditation session is over mindfulness practitioners will re-engage their socially-constructed, autobiographical narratives to reduce uncertainty associated with unresolved conflicts and make meaning out of the adversity in their lives (Olivares 2010). As the result of the decentering and psychological flexibility afforded by mindfulness practice, when practitioners return to the narrative-evaluative mode from the state of mindfulness, adaptive reappraisals of stressors are likely to emerge from conscious reflection on life circumstances or spontaneous insight. In this way, mindfulness training may facilitate traditional cognitive restructuring techniques in CBT that aim to help clients to adaptively reappraise their experience to reduce distress and self-destructive behaviors. Indeed, some CBT scientist-practitioners consider distancing or gaining objectivity toward thoughts (a process similar to decentering via mindfulness) to be a necessary step before an individual can successfully consider alternative appraisals during restructuring (Hofmann et al. 2012). As an exemplar of this therapeutic approach, Mindfulness-Oriented Recovery Enhancement involves a mindful reappraisal technique, in which clients are explicitly taught mindfulness skills in tandem with positive reappraisal techniques (Garland 2013). Clients are instructed to oscillate between mindfully disengaging from negative appraisals through mindfulness meditation and subsequently generating positive reappraisals through a Socratic process of inquiry that involves reframing the stressor in an adaptive manner and/or contemplating how facing the stressful situation can be a potential source of meaning or personal growth. In a RCT, this technique has been shown to enhance positive reappraisal ability (Garland et al. 2014b), yet more research is needed to explore the therapeutic outcomes of mindful reappraisal.

Beyond their clinical implications, results from the present study suggest that mindfulness and reappraisal are mutually-occurring and possibly mutually-reinforcing 
positive psychological processes that develop over time during the process of meditation training. At first glance, it may seem paradoxical that adopting a non-discursive, nonevaluative state of mind might promote a discursive process of making positive re-evaluations of life experience, but this paradox may reflect the fundamental and ubiquitous need of the human psyche for acceptance and change.

Acknowledgments During the preparation of this manuscript, E.L.G. was supported by National Institutes of Health grant number DA037005 and LGK was supported by Grant Number HL119977.

\section{Compliance with ethical standards}

Conflict of Interest Eric L. Garland, Laura G. Kiken, Kim Faurot, Olafur Palsson, and Susan A. Gaylord declare that they have no conflict of interest.

Informed Consent All procedures followed were in accordance with the ethical standards of the responsible committee on human experimentation and informed consent was obtained from each participant.

Animal Rights No animal studies were carried out by the authors for this article.

\section{References}

Bentler, P. M. (1990). Comparative fit indexes in structural models. Psychological Bulletin, 107(2), 238-246.

Bollen, K. A., \& Curran, P. J. (2004). Autoregressive latent trajectory (ALT) models a synthesis of two traditions. Sociological Methods \& Research, 32(3), 336-383.

Brown, K. W., \& Ryan, R. M. (2003). The benefits of being present: Mindfulness and its role in psychological well-being. Journal of Personality and Social Psychology, 84, 822-848.

Browne, M. W., \& Cudeck, R. (1993). Alternative ways of assessing model fit. SAGE Focus Editions, 154, 136.

Desrosiers, A., Vine, V., Klemanski, D. H., \& Nolen-Hoeksema, S. (2013). Mindfulness and emotion regulation in depression and anxiety: Common and distinct mechanisms of action. Depression and Anxiety, 30, 654-661.

Garland, E. L., Fredrickson, B. L., Kring, A. M., Johnson, D. P., Meyer, P. S., \& Penn, D. L. (2010). Upward spirals of positive emotions counter downward spirals of negativity: Insights from the broaden-and-build theory and affective neuroscience on the treatment of emotion dysfunctions and deficits in psychopathology. Clinical Psychology Review, 30, 849-864.

Garland, E. L., Gaylord, S. A., \& Fredrickson, B. L. (2011). Positive reappraisal coping mediates the stress-reductive effect of mindfulness: An upward spiral process. Mindfulness, 2, 59-67.

Garland, E. L. (2013). Mindfulness-Oriented Recovery Enhancement for addiction, stress, and pain. Washington, D.C.: NASW Press.

Garland, E. L., Froeliger, B., \& Howard, M. O. (2014a). Effects of Mindfulness-Oriented Recovery Enhancement on reward responsiveness and opioid cue-reactivity. Psychopharmacology (Berl), 231(16), 3229-3238.

Garland, E. L., Manusov, E. G., Froeliger, B., Kelly, A., Williams, J. M., \& Howard, M. O. (2014b). Mindfulness-Oriented Recovery Enhancement for chronic pain and prescription opioid misuse: Results from an early-stage randomized controlled trial. Journal of Consulting and Clinical Psychology, 82(3), 448.
Garland, E. L., Roberts-Lewis, A., Kelley, K., Tronnier, C., \& Hanley, A. (2014c). Cognitive and affective mechanisms linking trait mindfulness to craving among individuals in addiction recovery. Substance Use and Misuse, 49(5), 525-535.

Garland, E. L., Farb, N. A., Goldin, P. R., \& Fredrickson, B. L. (2015a). Mindfulness broadens awareness and builds eudaimonic meaning: A process model of mindful positive emotion regulation. Psychological Inquiry, 26(4), 293-314.

Garland, E. L., Farb, N. A., Goldin, P. R., \& Fredrickson, B. L. (2015b). The Mindfulness-to-Meaning Theory: Extensions, applications, and challenges at the attention-appraisal-emotion interface. Psychological Inquiry, 26(4), 377-387.

Garland, E. L., Froeliger, B., \& Howard, M. O. (2015c). Neurophysiological evidence for remediation of reward processing deficits in chronic pain and opioid misuse following treatment with Mindfulness-Oriented Recovery Enhancement: Exploratory ERP findings from a pilot RCT. Journal of Behavioral Medicine, $38(2), 327-336$.

Garland, E. L., Geschwind, N., Peeters, F., \& Wichers, M. (2015d). Mindfulness training promotes upward spirals of positive affect and cognition: Multilevel and autoregressive latent trajectory modeling analyses. Frontiers in Psychology. doi:10.3389/fpsyg. 2015.00015.

Garland, E. L., Hanley, A., Farb, N. A., \& Froeliger, B. (2015e). State mindfulness during meditation predicts enhanced cognitive reappraisal. Mindfulness, 6, 234-242.

Garnefski, N., \& Kraaij, V. (2007). The cognitive emotion regulation questionnaire: Psychometric features and prospective relationships with depression and anxiety in adults. European Journal of Psychological Assessment, 23, 141-149.

Geschwind, N., Peeters, F., Drukker, M., van Os, J., \& Wichers, M. (2011). Mindfulness training increases momentary positive emotions and reward experience in adults vulnerable to depression: A randomized controlled trial. Journal of Consulting and Clinical Psychology, 79(5), 618.

Goyal, M., Singh, S., Sibinga, E. M., Gould, N. F., RowlandSeymour, A., Sharma, R., et al. (2014). Meditation programs for psychological stress and well-being: a systematic review and meta-analysis. JAMA Internal Medicine, 174(3), $357-368$.

Hanley, A. W., \& Garland, E. L. (2014). Dispositional mindfulness co-varies with self-reported positive reappraisal. Personality and Individual Differences, 66, 146-152.

Hanley, A. W., Warner, A., \& Garland, E. L. (2015). Associations between mindfulness, psychological well-being, and subjective well-being with respect to contemplative practice. Journal of Happiness Studies, 16, 1423-1426.

Hofmann, S. G., Sawyer, A. T., Fang, A., \& Asnaani, A. (2012). Emotion dysregulation model of mood and anxiety disorders. Depression and Anxiety, 29, 409-416.

Hofmann, S. G., Sawyer, A. T., Witt, A., \& Oh, D. (2010). The effect of mindfulness-based therapy on anxiety and depression: A meta-analytic review. Journal of Consulting and Clinical Psychology, 78, 169-183.

Hölzel, B. K., Lazar, S. W., Gard, T., Schuman-Olivier, Z., Vago, D. R., \& Ott, U. (2011). How does mindfulness meditation work? Proposing mechanisms of action from a conceptual and neural perspective. Perspectives on Psychological Science, 6(6), $537-559$.

Huston, D. C., Garland, E. L., \& Farb, N. A. (2011). Mechanisms of mindfulness in communication training. Journal of Applied Communication Research, 39(4), 406-421.

Jones, S. M., \& Hansen, W. (2015). The impact of mindfulness on supportive communication skills: Three exploratory studies. Mindfulness, 6, 1115-1128.

Kabat-Zinn, J. (1990). Full Catastrophe living. NY: Delacorte Press. 
Khoury, B., Lecomte, T., Fortin, G., Masse, M., Therien, P., Bouchard, V., et al. (2013). Mindfulness-based therapy: A comprehensive meta-analysis. Clinical Psychology Review, 33, 763-771.

Kiken, L. G., Garland, E. L., Bluth, K., Palsson, O. S., \& Gaylord, S. A. (2015). From a state to a trait: Trajectories of state mindfulness in meditation during intervention predict changes in trait mindfulness. Personality and Individual Differences, 81, 41-46.

Kline, R. B. (1998). Principles and practice of structural equation modeling. NY: Guilford Press.

Lau, M. A., Bishop, S. R., Segal, Z. V., Buis, T., Anderson, N. D., Carlson, L., et al. (2006). The Toronto Mindfulness Scale: Development and validation. Journal of Clinical Psychology, 62, 1445-1467.

Lazarus, R., \& Folkman, S. (1984). Stress, appraisal, and coping. New York: Springer.
Olivares, O. J. (2010). Meaning making, uncertainty reduction, and the functions of autobiographical memory: A relational framework. Review of General Psychology, 14, 204-211.

Opialla, S., Lutz, J., Scherpiet, S., Hittmeyer, A., Jäncke, L., Rufer, M., et al. (2014). Neural circuits of emotion regulation: A comparison of mindfulness-based and cognitive reappraisal strategies. European Archives of Psychiatry and Clinical Neuroscience, 265(1), 45-55.

Ryan, R. M., \& Deci, E. L. (2001). On happiness and human potentials: A review of research on hedonic and eudaimonic well-being. Annual Review of Psychology, 52(1), 141-166.

Ryff, C. D., \& Singer, B. H. (2008). Know thyself and become what you are: A eudaimonic approach to psychological well-being. Journal of Happiness Studies, 9, 13-39.

Troy, A., Shallcross, A., Davis, T., \& Mauss, I. (2013). History of mindfulness-based cognitive therapy is associated with increased cognitive reappraisal ability. Mindfulness, 4, 213-222. 